Recepción: 01 / 10/ 2018

Aceptación: 15 / 11 / 2018

Publicación: 11 / 12 / 2018
Ciencias de la Salud

Artículo Científico

\title{
Materiales tóxicos y su incidencia en el ambiente hospitalario
}

Toxic materials and their incidence in the hospital environment

\section{Materiais tóxicos e sua incidência no ambiente hospitalar}

\author{
Claudia V. Romero-Castro ${ }^{\mathrm{I}}$ \\ claudia.castro@gmail.com \\ Mónica M. Solórzano-Vélez II \\ monicamvelez@gmail.com \\ Adriana I. Navarrete-Ríos III \\ adriana.nrios@gmail.com
}

\author{
Karen X. Córdova-Chinga IV \\ karencordova.x@gmail.com \\ Iván A. Jaramillo-Chávez ${ }^{\mathrm{V}}$ \\ ivan.chavez@gmail.com \\ Alejandra I. Navarrete-Ríos VI \\ alejandranrios@gmail.com
}

Correspondencia: claudia.castro@gmail.com

I. Médico Cirujano. Administradora técnica del establecimiento de salud Cheve Arriba, Manabí, Pedernales, Ecuador.

II. Médico General. Centro de salud Flavio Alfaro, Chone, Ecuador.

III. Médico Cirujano. Ministerio de Salud Pública, Manta, Ecuador.

IV. Médico General de planta. Empresa exportadora Seafman S.A, Manta, Ecuador.

v. Médico Residente. Servicio de Medicina Interna Hospital de especialidades, Portoviejo, Ecuador.

VI. Médico Cirujano. Ministerio de Salud Pública, Manta, Ecuador. 
Claudia V. Romero-Castro; Mónica M. Solórzano-Vélez; Adriana I. Navarrete-Ríos; Karen X. Córdova-Chinga; Iván A. Jaramillo-Chávez; Alejandra I. Navarrete-Ríos

\section{Resumen}

Las intoxicaciones son un problema de salud pública y una de las principales causas de ingresos en las salas de emergencias a nivel nacional e internacional. La inadecuada recolección, transporte, almacenamiento y disposición final de los desechos hospitalarios puede provocar daños físicos serios e infecciones graves al personal que labora en los hospitales, a los pacientes y a la comunidad en general. Además, existe la posibilidad de que la exposición prolongada a contaminantes infecciosos y/o tóxicos, aunque sea a niveles bajos, pueda incrementar la susceptibilidad del personal de salud y de los pacientes, para desarrollar enfermedades preexistentes. Todo este riesgo infeccioso y químico puede ser controlado mediante un manejo adecuado de los desechos hospitalarios. Esta investigación tiene como objetivo general analizar los materiales tóxicos y su incidencia en el ambiente hospitalario. Se realizó una investigación exploratoria, descriptiva, transversal y de observación dirigida, para determinar la incidencia de las intoxicaciones. Los resultados se basaron en un análisis sobre el manejo de los materiales tóxicos y la incidencia d ellos mismo en los centros de salud. Como conclusión se obtuvo que se deben cumplir las normas internacionales de manejo, traslado y eliminación de los materiales tóxicos con el fin de disminuir los factores de riesgos de contaminación en las poblaciones.

Palabras claves: Materiales tóxicos, intoxicaciones, un riesgo químico, infección. 


\begin{abstract}
Poisonings are a public health problem and one of the main causes of income in emergency rooms nationally and internationally. Inadequate collection, transportation, storage and final disposal of hospital waste can cause serious physical harm and serious infections to staff working in hospitals, patients and the community in general. In addition, there is the possibility that prolonged exposure to infectious and / or toxic contaminants, even at low levels, may increase the susceptibility of health personnel and patients to develop pre-existing diseases. All this infectious and chemical risk can be controlled by proper management of hospital waste. The general objective of this research is to analyze the toxic materials and their incidence in the hospital environment. An exploratory, descriptive, crosssectional and directed observation research was carried out to determine the incidence of intoxications. The results were based on an analysis of the handling of toxic materials and the incidence of them in health centers. As a conclusion it was obtained that the international norms of handling, transfer and elimination of the toxic materials must be fulfilled in order to reduce the risk factors of pollution in the populations.
\end{abstract}

Keys words: Toxic materials, poisonings, a chemical risk, infection. 
Claudia V. Romero-Castro; Mónica M. Solórzano-Vélez; Adriana I. Navarrete-Ríos; Karen X. Córdova-Chinga; Iván A. Jaramillo-Chávez; Alejandra I. Navarrete-Ríos

\section{Introducción.}

El crecimiento y desarrollo de la sociedad ha venido de la mano de grandes cambios en su entorno. Estos cambios traen como consecuencia muchos factores de riesgo producto del avance de la humanidad con el fin de garantizar las necesidades de su entorno. El alto rango de amenazas existentes se combina con los procesos y expresiones diversas de la vulnerabilidad, para crear muy variados contextos de riesgo en el espacio urbano, en donde estos varían de acuerdo a su tamaño y complejidad (Lavell, Desastres urbanos: una visión global., 2000).

Los espacios urbanos juegan un papel importante en el procesamiento y manejo de desechos producto del desarrollo de las sociedades. Las infraestructuras de drenaje, de distribución de aguas y descarga de aguas residuales, los puentes, mercados y edificios públicos, las escuelas y hospitales, entre otros, sufren en muchos casos años de olvido, sin reparación o renovación (Lavell, Gestión de riesgos ambientales urbanos. Red de Estudios Sociales en Prevención de Desastres en América Latina, 1999).

Este crecimiento poblacional en zonas no estipuladas para tales construcciones ha producido que los alimentos tengan un alto riesgo de contaminación por la insalubridad presentes en los sitios. Los riesgos son: por microorganismos patógenos que contaminan los alimentos (falta de higiene en su manejo), por sustancias tóxicas naturales, contenidas en los alimentos, por contaminantes ambientales que afectan los alimentos (plaguicidas, metales, sales, componentes de envases) y por aditivos alimentarios, siempre que sean autorizados y estén debidamente declarados (SchmidtHebbel, 2018). 
Por los cuales los contaminantes ambientales juegan un papel importante en la salud de la población y esta a su vez esta relacionada con las políticas gubernamentales aplicadas por el estado y de la aceptación de la sociedad. Unos de esos contaminantes ambientales que más producen daños en la calidad de vida son las sustancias toxicas. Los vertimientos de sustancias toxicas al medio ambiente, ha sido una problemática que desde hace algunas décadas ha llamado la atención de organismos gubernamentales. Numerosas técnicas se han empleado con el fin de minimizar el impacto que estas causan a los ecosistemas (Garzón-Jiménez, 2008).

Es importante resaltar que las sustancias tóxicas producen efectos nocivos cuando penetra en el organismo tales como dolor de cabeza o nauseas, convulsiones o coma o en casos más graves la persona puede morir (MSAL, 2018). También MSAL (2018) comentan que todos los productos químicos pueden actuar como toxico si la cantidad presente en el cuerpo es suficiente ya que algunos son nocivos con cantidades pequeñas mientras que otros lo son con grandes cantidades (detergentes domésticos).

Es por ello que es necesario la implementación de identificación de las sustancias tóxicas para así poder manejarlos con la precaución debida. Las actividades que se requieren para controlar una emergencia con materiales peligrosos se basan en la identificación de los materiales o sustancias peligrosas involucradas. La facilidad y rapidez para hacerlo varía considerablemente a diferencia de que no se tenga ningún sistema de identificación (Díaz, 2011). Esto se puede ver en la Figura 1. 
Claudia V. Romero-Castro; Mónica M. Solórzano-Vélez; Adriana I. Navarrete-Ríos; Karen X. Córdova-Chinga; Iván A. Jaramillo-Chávez; Alejandra I. Navarrete-Ríos

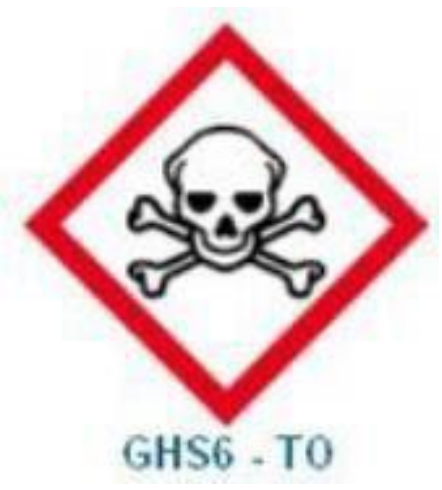

Figura 1. Identificación de las sustancias toxicas

Fuente: (UNAVARRA, 2018)

Existen varias formas de como estas sustancias toxicas pueden entrar en el organismo d las personas que tengan contacto con ellas, las cuales se pueden identificar mediante la Figura 2.

$\checkmark \quad$ VÍA RESPIRATORIA. Es la vía de penetración de sustancias tóxicas más importantes en el medio ambiente de trabajo, ya que con el aire que respiramos pueden penetrar en nuestro organismo polvos, humos, aerosoles, gases, etc.

$\checkmark \quad$ VÍA DIGESTIVA. Es la vía de penetración a través de la boca, el esófago, el estómago y los intestinos. También hemos de considerar la posible ingestión de contaminantes disueltos en mucosidades del sistema respiratorio.

$\checkmark \quad$ VÍA PARENTERAL. Es la vía de penetración del contaminante en el cuerpo a través de llagas, heridas, etc.

$\checkmark \quad$ VÍA DÉRMICA. Es la vía de penetración de muchas sustancias que son capaces de atravesar la piel, sin causar erosiones o alteraciones notables, e incorporarse a la sangre, para posteriormente ser distribuidas por todo el cuerpo. (UNAVARRA, 2018, p. 6) 


\section{VÍA RESPIRATORIA}

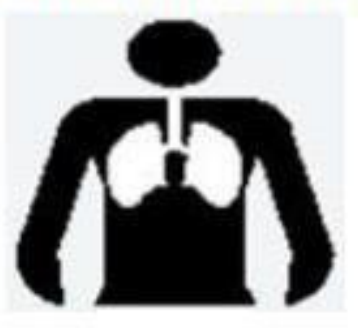

VÍA PARENTERAL

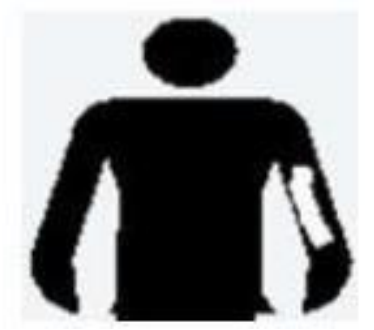

VÍA DIGESTIVA

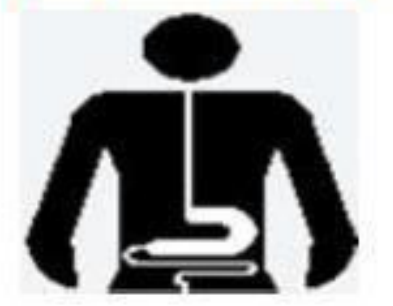

VÍA DÉRMICA

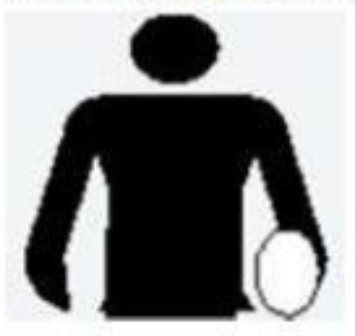

Figura 2. Vías de entrada del contaminante al cuerpo

Fuente: (UNAVARRA, 2018)

Por lo cual es importante considerar el manejo y traslado de los productos tóxicos. Los productos peligrosos generalmente se transportan y almacenan en grandes cantidades. Una fuga accidental de estos productos representa un riesgo potencial para las personas y el ambiente. El accidente se puede tratar más rápidamente cuando se identifica y caracteriza el producto peligroso (Haddad, Serpa, \& Arias, 2018)

Ahora poder trasladar estas circunstancias dentro de los centros de salud es importante. El manejo y la disposición final de los desechos hospitalarios ha sido objeto de atención en todo el mundo. La Organización Mundial de la Salud (OMS) ha realizado paneles de expertos para analizar la materia y ha difundido recomendaciones específicas para países en vías de desarrollo. Frente a la postura de la OMS y de diversas agencias gubernamentales que promueven un tratamiento agresivo de los residuos biológicos, existen también posiciones divergentes. Tomando como base los riesgos 
Claudia V. Romero-Castro; Mónica M. Solórzano-Vélez; Adriana I. Navarrete-Ríos; Karen X. Córdova-Chinga; Iván A. Jaramillo-Chávez; Alejandra I. Navarrete-Ríos

epidemiológicos conocidos, hay quienes postulan que la gran mayoría de los residuos generados por las instituciones de salud son asimilables a los desechos domésticos, y que por lo tanto, no merecen un manejo especial, salvo casos específicos como los elementos radioactivos, los agentes citotóxicos, y otros de connotaciones especiales como los restos humanos identificables a simple vista. Este contexto de controversia, y el impacto económico que las decisiones en esta materia pueden tener para el sistema público de salud, justifican la evaluación objetiva de los riesgos reales asociados a los desechos hospitalarios para la población, y a la adopción de medidas de protección efectivas en los casos en los que la evidencia científica y epidemiológica lo sugiera.

Por lo tanto, esta investigación tiene como objetivo general analizar los materiales tóxicos y su incidencia en el ambiente hospitalario. La metodología empleada se baso en ¿investigación descriptiva, documental de campo y exploratoria explicativa tomando en consideración ciertas fuentes bibliográficas.

\section{Método.}

La metodología utilizada para esta investigación se basó en los análisis sobre los diferentes materiales de desecho, las características de los materiales tóxicos, la clasificación de los mismos, el proceso de almacenaje de los materiales tóxicos y la incidencia de estos dentro de los centros de salud. Esto se basó realizando una investigación descriptiva, en documentación de campo y en la información descriptiva exploratoria tomando en cuanto ciertos medios como lo son los sitios web, libros, revistas y entrevistas a personas que han padecido esta enfermedad

\section{Desarrollo.}

\section{Manejo de los materiales tóxicos}


El manejo de los materiales tóxicos debe realizarse bajo los estándares de las normas internacionales de protección del medio ambiente y ecosistema. Las actividades generadas deben ser documentados con el fin de poder determinar la forma, aparición, manejo y traslado de los residuos tóxicos. Para los registros de la revisión del sistema de gestión ambiental se puede incluir copias de la agenda, de reuniones, listas de asistencia, presentaciones, documentación y decisiones de la gerencia que serán registradas en actas, informes, resúmenes, memorándums o sistemas de seguimiento (Díaz Espinel \& Ramos Rubianes, 2012). En la Figura 3 se puede detallar el flujograma del proceso de implementación de la norma ISO 14001.

Cumpliendo estas normativas de gestión medioambiental entonces es necesario poder realizar un plan de análisis que incluye la descripción de los procedimientos de análisis, los equipos requeridos, métodos de calibración, calidad y exactitud de los resultados obtenidos por el método de análisis (Soto \& Guerrero, 1996).

Según Soto y Guerrero (1996) existen tres metas principales del procedimiento de muestreo y análisis. Estos son: identificar preliminarmente la peligrosidad inherente del material, analizar detalladamente las características fisicoquímicas del residuo para manejarlo efectivamente y encontrar la principal característica peligrosa que facilite la identificación del cargamento manejado

Después de este análisis y comprensión de la identificación fisicoquímica del material toxico entonces se procede a realizar un buena almacenado de las sustancias siguiendo las pautas de las normas internacionales. Estas son: 
Claudia V. Romero-Castro; Mónica M. Solórzano-Vélez; Adriana I. Navarrete-Ríos; Karen X. Córdova-Chinga; Iván A. Jaramillo-Chávez; Alejandra I. Navarrete-Ríos

$\checkmark \quad$ Almacene los materiales tóxicos de manera para prevenir la contaminación de comida, equipo, utensilios, linos, artículos designados "single service" o solo servicio y artículos no reutilizables.

$\checkmark \quad$ No se permite almacenar los materiales tóxicos arriba de o a la par de la comida o de superficies que tienen contacto con la comida, no importa el empaquetado.

$\checkmark \quad$ Almacene los agentes de limpieza aparte de los insecticidas.

$\checkmark \quad$ Almacene abastos de primeros auxilios o los remedios personales, de una manera que prevenga contaminación de comidas o superficies que tienen contacto con la comida. (Pública, 2018, p. 1)

Según UNAVARRA (2018) también existen normativas de almacenado de sustancias químicas, incluyendo las sustancias tóxicas.

$\checkmark \quad$ Comprobar que los productos están adecuadamente etiquetados. En la etiqueta es donde está la primera información sobre los riesgos de los productos químicos en los pictogramas de riesgo y las frases $\mathrm{H}$, lo cual es una primera información útil para saber cómo hay que almacenar los productos.

$\checkmark \quad$ Disponer de su ficha de datos de seguridad (FDS). Llevar un registro actualizado de la recepción de los productos que permita evitar su envejecimiento.

$\checkmark \quad$ Agrupar y clasificar los productos por su riesgo respetando las restricciones de almacenamientos, así como las cantidades máximas recomendadas. Las separaciones podrán efectuarse, en función del tamaño del almacén, bien por el sistema de islas, bien por el de estanterías. 
$\checkmark \quad$ En el caso de uso de estanterías, estrados, soportes de madera estas serán macizas y de un espesor mínimo de 25mm.

$\checkmark \quad$ Limitar el stock de productos y almacenar sistemáticamente la mínima cantidad posible.

$\checkmark \quad$ Disponer en el área de trabajo solamente de los productos que se vayan a utilizar y mantener el resto de los productos en un área de almacenamiento.

$\checkmark \quad$ Los almacenes de productos tóxicos en laboratorios estarán dotados de ventilación forzada, que tengan salida al exterior.

$\checkmark \quad$ Implantar procedimientos de orden y limpieza y comprobar que son seguidos por los trabajadores.

$\checkmark \quad$ Planificar las emergencias tales como la actuación en caso de una salpicadura, un derrame o rotura de un envase, un incendio, etc.

$\checkmark \quad$ Formar e informar a los trabajadores sobre los riesgos del almacenamiento de productos, como prevenirlos y como protegerse.

$\checkmark \quad$ Prohibido fumar.

$\checkmark \quad$ Prohibido utilizar llamas abiertas o fuentes de ignición. (p. 12)

Cumplir con estas pautas puede generar un buen ambiente de trabajo. Un manejo deficiente de los desechos peligrosos puede ser la causa de situaciones de deterioro ambiental que pone en riesgo la salud de aquellos sectores que, directa o indirectamente, están en contacto con desechos peligrosos (Colmenares, 2014). Tal es el caso de los productos desechados por las industrias farmacéuticas las cuales cumplen un protocolo del manejo y eliminación con el fin de que dichos productos no lleguen a las manos de los consumidores. Esto se puede reflejar en la Figura 4. 
Claudia V. Romero-Castro; Mónica M. Solórzano-Vélez; Adriana I. Navarrete-Ríos; Karen X. Córdova-Chinga; Iván A. Jaramillo-Chávez; Alejandra I. Navarrete-Ríos

Extrapolar estas situaciones a los países trae consigo el foco de contaminación que va a depender del grado de cultura de los entes gubernamentales y de la sociedad. Las precarias condiciones de vida de la inmensa mayoría de los países del Tercer Mundo amenazan agravarse en el corto plazo ante las masivas exportaciones de millones de toneladas de residuos peligrosos provenientes de países industrializados (Sánchez, 1990).

En el ecuador existen leyes y reglamentos nacionales vigentes y convenios internacionales que permiten el riguroso manejo de los materiales tóxicos. Según la norma técnica ecuatoriana NTE INEN 2266:2013 en su segunda revisión refleja que:

Todas las personas naturales o jurídicas que almacenen, manejen y transporten materiales peligrosos deben garantizar que cuando se necesite cargar o descargar la totalidad o parte de su contenido, el transportista y el usuario deben instalar señalización o vallas reflectivas de alta intensidad o grado diamante con la identificación del material peligroso, que aíslen la operación, con todas las medidas de seguridad necesarias. (INEN, 2018, p. 10) 


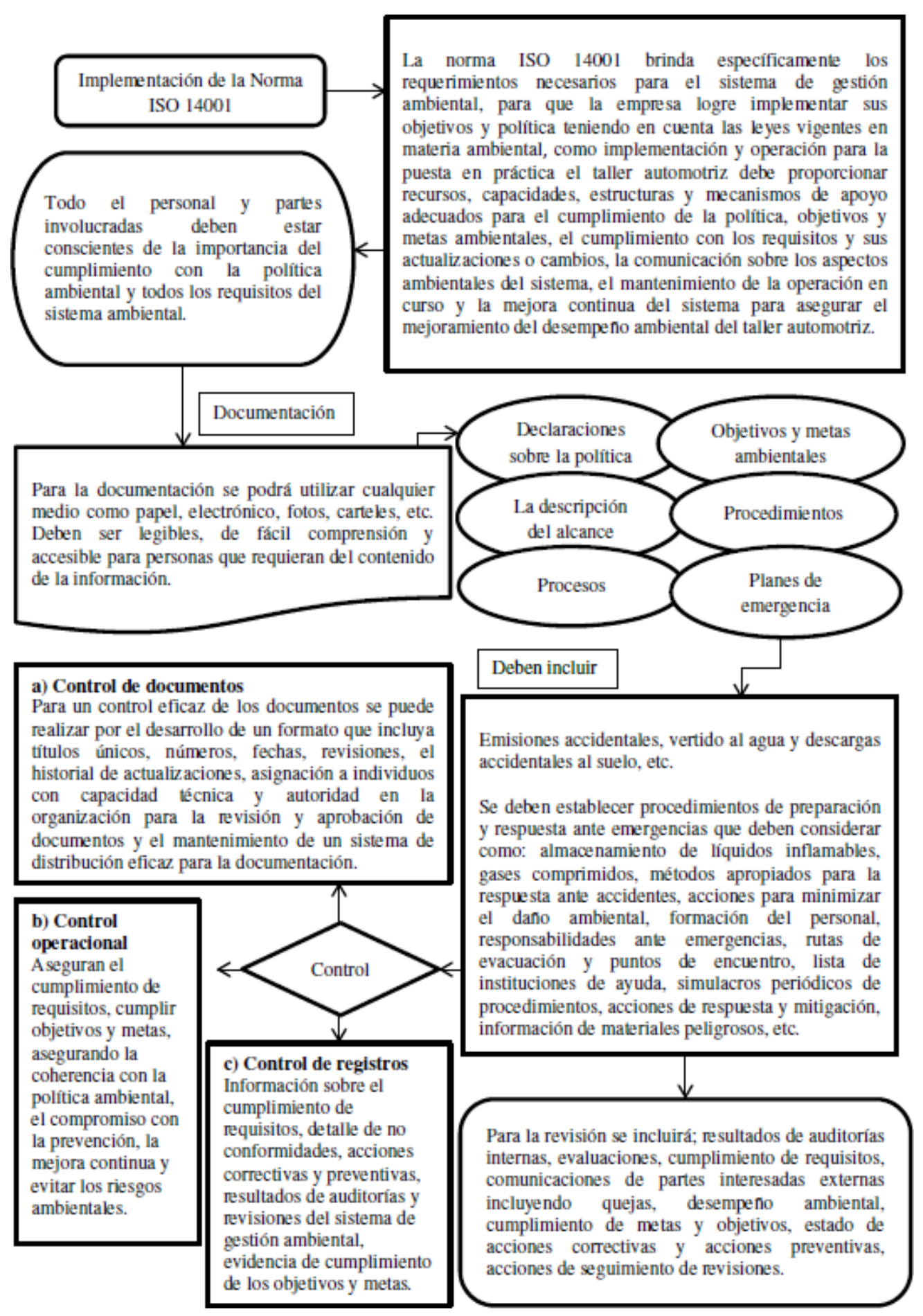

Figura 3. Flujograma del proceso de implementación de la norma ISO 14001

Fuente: (Díaz Espinel \& Ramos Rubianes, 2012) 
Claudia V. Romero-Castro; Mónica M. Solórzano-Vélez; Adriana I. Navarrete-Ríos; Karen X. Córdova-Chinga; Iván A. Jaramillo-Chávez; Alejandra I. Navarrete-Ríos

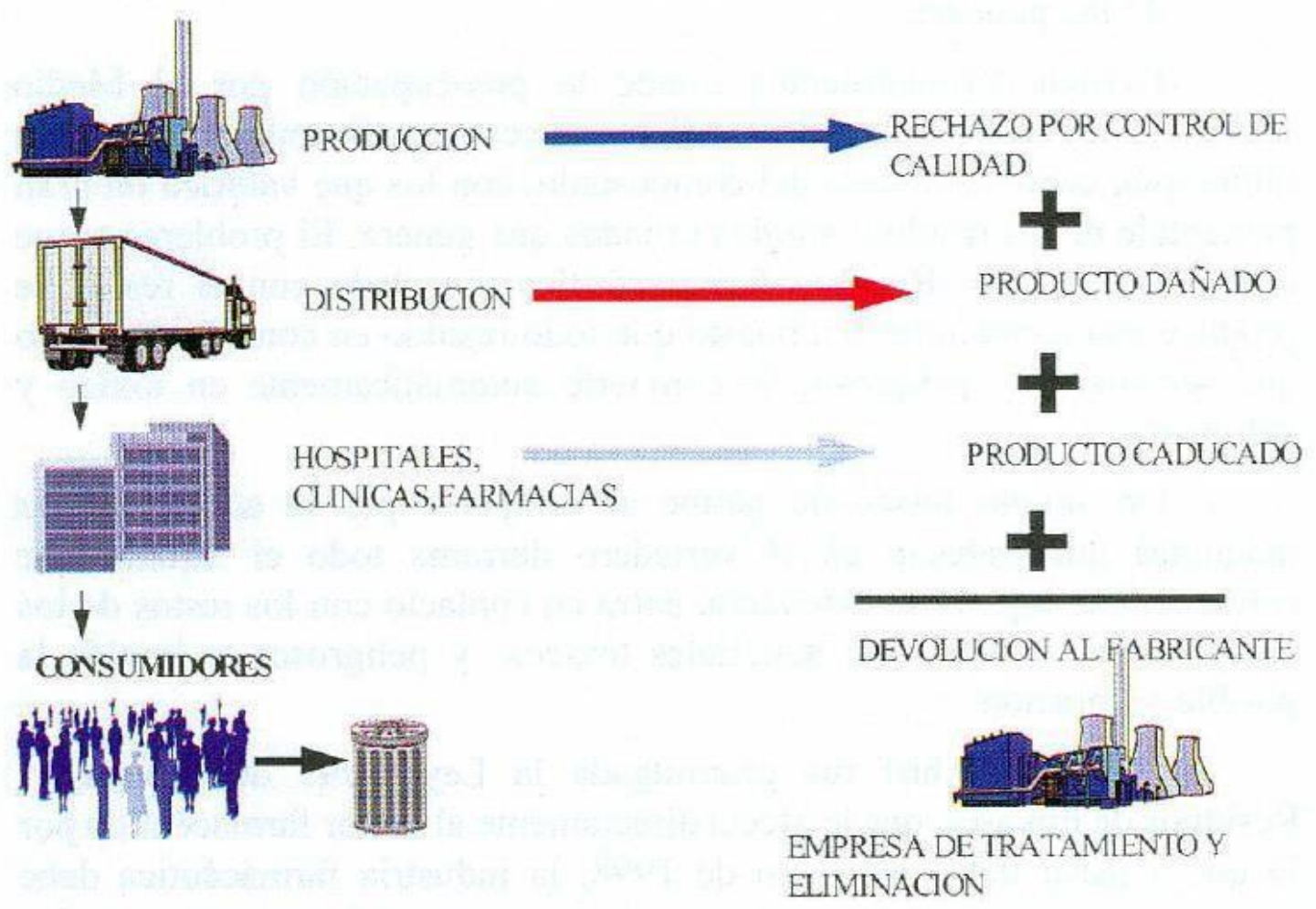

Figura 4. Ciclo de vida de los productos farmacéuticos

Fuente: (Cabrejas \& González, 1998)

\section{Los materiales tóxicos en los ambientes de salud}

Estos últimos incluyen además los provenientes de clínicas y consultas médicas, de centros ambulatorios, de clínicas dentales, de laboratorios, de centros de investigación, de los cuidados de salud domiciliaria (pacientes diabéticos, tratamientos ambulatorios de cuadros agudos por vía intravenosa o intramuscular, etc), de oficinas donde se practica atención de enfermería, y de centros de diálisis, entre otros. Contribuyen también a acrecentar tales riesgos y dificultades la heterogeneidad de su composición, la presencia frecuente de objetos cortopunzantes y la presencia eventual de cantidades menores de sustancias tóxicas, inflamables y radiactivas de baja intensidad (Rodríguez Sordía, 2000). 
Nuevamente aquí es preciso determinar la acción de las normas internacionales sobre la gestión medioambiental ya que los materiales tóxicos pueden generar problemas no solo al ecosistema sino también a la sociedad.

La adecuada gestión de los residuos en el laboratorio no es solamente una necesidad con el objeto de mejorar las condiciones de trabajo, sino que constituye una pieza fundamental en la aplicación de criterios de calidad y gestión ambiental en el laboratorio, siendo también, obviamente, una de las exigencias de aplicación de las buenas prácticas (BPL). (Carrera, 2005, p. 2)

Dentro de los centros de salud debe haber políticas que permitan el mejor manejo dentro de los residuos tóxicos. Según Cifuentes y Iglesias (2008) se plantean de la siguiente manera

$\checkmark \quad$ Respetar el ambiente protegiéndolo y garantizando la armonía entre sus actividades y el ambiente.

$\checkmark \quad$ Cumplir con la normatividad ambiental nacional y cuando ésta no exista en temas involucrados con sus actividades, tomar en cuenta la normatividad internacional.

$\checkmark \quad$ Mantener comunicación fluida con el sector competente en cuanto a la relación entre sus operaciones y los aspectos ambientales.

$\checkmark \quad$ Informar a la comunidad y grupos de interés (pacientes, trabajadores, proveedores, contratistas) sobre sus actividades y su política ambiental para que sean cumplidas por éstos.

$\checkmark \quad$ Mantener capacitados a sus trabajadores para que la política ambiental pueda ser cumplida. 
Claudia V. Romero-Castro; Mónica M. Solórzano-Vélez; Adriana I. Navarrete-Ríos; Karen X. Córdova-Chinga; Iván A. Jaramillo-Chávez; Alejandra I. Navarrete-Ríos

$\checkmark \quad$ Asegurar que cada elemento o cambio de los procedimientos hospitalarios

incluya las normas y política ambiental.

$\checkmark \quad$ Monitorear los componentes de su gestión ambiental para garantizar su buen

funcionamiento y el estado saludable del ambiente. (p. 24)

Para poder cumplir con estas políticas también deben generare dentro de los centros de salud actividades pedagógicas que permitan a los empleados conocer el manejo de los materiales tóxicos. Los empleados presentan poco conocimiento y mala prácticas frente al manejo de residuos, lo cual es independiente del sexo, la edad, el área de trabajo y el tiempo de servicio; esto implica que el problema está focalizado como un tema generalizado de las instituciones (Quinto-Mosquera, Pérez, \& Arias, 2013)

Para que estos empleados puedan tener conocimiento sobre como es el funcionamiento del manejo de los materiales tóxico es necesario poder clasificar los desechos hospitalarios según la Organización Mundial de la Salud. Esta clasificación se basa en residuos generales, residuos patológicos, residuos radioactivos, residuos químicos, residuos infecciosos, residuos punzocortantes y residuos farmacéuticos (Monge, 1997).

Uno de los casos de manejo que requiere de mucha precaución son los procedentes de centro de salud de alta complejidad. Dentro de estos se encuentra los residuos cortopunzantes.

La costumbre de eliminar los residuos cortopunzantes en caja rígida junto con los residuos sólidos urbanos, es una acción que reduce el riesgo para los manipuladores de los residuos (personal médico y personal de aseo), al no contar el país con un sistema que asegure la adecuada disposición de residuos urbanos (vertederos ilegales y sellado diario insuficiente), en aquellas 
localidades en que la actividad de rastrojear basura doméstica persiste, esta modalidad de disposición puede resultar poco segura. (Neveu y Matus, 2007, p. 894)

También existe otros tipos de desechos como los son los infecciosos. El manejo inadecuado de los desechos infecciosos y las consecuencias obligan a los profesionales de la salud, a estar debidamente informados, concientizados, para tomar las acciones que correspondan y no ser un eslabón más en la diseminación de las infecciones (Muñoz Falconi, 2015)

Por ello es siempre necesario crear un manual de procedimiento que pueda integrar todas las características de los materiales tóxicos basándose en las normas internacionales de manejo del mismo y de la gestión medioambiental. Según un manual debe tener estos objetivos:

$\checkmark \quad$ Incremento de la seguridad. Al establecer el programa se brinda mayor seguridad al personal, pacientes y visitantes de estos establecimientos. Con un manejo adecuado de los residuos se corta la cadena de transmisión de los gérmenes patógenos contenidos en los desechos y se evita la aparición de agentes transmisores dentro y fuera del hospital.

$\checkmark \quad$ Reducción del impacto ambiental. Se reduce la cantidad de residuos peligrosos existentes en la ciudad y se mejora la imagen de los establecimientos de salud. Junto con los departamentos o empresas de aseo de los municipios se promueve la correcta transportación y disposición final de los desechos infecciosos, minimizando el impacto que éstos pueden ocasionar al ambiente.

$\checkmark \quad$ Optimización de los costos. El manejo desorganizado de los residuos, particularmente de los infecciosos, incrementa el número de infecciones intrahospitalarias y el ausentismo del personal. Ambas situaciones, elevan los gastos de atención médica y los costos 
Claudia V. Romero-Castro; Mónica M. Solórzano-Vélez; Adriana I. Navarrete-Ríos; Karen X. Córdova-Chinga; Iván A. Jaramillo-Chávez; Alejandra I. Navarrete-Ríos

por días no laborados. Al separar los desechos infecciosos, que constituyen sólo el 10\% del total, disminuyen los costos de tratamiento ya que el $90 \%$ de residuos no lo necesitan. Si los programas de manejo logran separar reciclables y venderlos, estos ingresos ayudan a cubrir los gastos que implica el manejo adecuado. (p. 2-3)

Oro tema fundamental relacionado con la gestión de residuos hospitalarios es la incineración. Pero ésta actividad produce dioxinas, que es una de las sustancias contaminantes más tóxicas y persistentes conocidas por la ciencia. La mayoría de los países industrializados insisten en implementar medidas de control complejas y costosas para capturar tales sustancias. Luego se debe realizar la disposición especial de los subproductos generados, por ejemplo, las cenizas de la combustión, por tratarse de residuos peligrosos.

\section{Discusión y conclusiones.}

El avance de la tecnología ha producido un incremento de desechos que producen una grave contaminación hacia el medioambiente. Es necesario poder clasificar los desechos variando su reacciones, propiedades fisicoquímicas y peligrosidad. Dentro de los más peligrosos se encuentran los materiales tóxicos las cuales pueden entrar en contacto por varias vías del organismo sino se tiene un buen manejo de los mismo. Es por ello que se deben implementar políticas que rijan el procesamiento y desecho de los mismos.

Los desechos tóxicos primeramente deben estar bien identificados para que los ciudadanos puedan tener el cuidado pertinente según sea el caso. El proceso de almacenaje debe cumplir con los estándares internacionales que permitan una buena manipulación. Es necesario que los entes 
gubernamentales fomenten una cultura del cuidado del medio ambiente y de la sociedad a través de taller informativos que cumplan con la orientación pertinente.

No existen condiciones de riesgo que puedan considerarse absolutas respecto a los desechos hospitalarios. Su nivel de riesgo tiene que ver principalmente con: La presencia de tipos específicos de desechos de alto riesgo. La gran mayoría de los desechos hospitalarios reconocidos tradicionalmente como desechos infecciosos son asimilables a los desechos domésticos en cuanto a su composición y riesgo, y por lo tanto no se recomienda que sean sometidos en forma sistemática a tratamiento de desinfección o esterilización.

\section{Referencias.}

Cabrejas, J. M., \& González, D. (1998). El residuo Farmacéutico. Monografías de la Real Academia Nacional de Farmacia.

Carrera, E. G. (2005). NTP 359: Seguridad en el laboratorio: gestión de residuos tóxicos y peligrosos en pequeñas cantidades. España.: Notas Técnicas Instituto Nacional de Seguridad e Higiene en el Trabajo. Ministerio de Trabajo y Asuntos Sociales.

Cifuentes, C., \& Iglesias, S. (2008). Gestión ambiental de residuos sólidos hospitalarios de Hospital Cayetano Heredia. Revista del Instituto de Investigación de la Facultad de Ingeniería Geológica, Minera, Metalurgica y Geográfica, 12(23), 27-39.

Colmenares, C. (2014). Bases para el manejo de sustancias químicas peligrosas, en laboratorios de docencia de la escuela de ingeniería química. Valencia - Venezuela: Centro de Investigaciones Ambientales de la Universidad de Carabobo (CIAUC).

Díaz Espinel, G. A., \& Ramos Rubianes, A. E. (2012). Elaboración de un manual de procedimientos para el manejo de desechos tóxicos y desarrollo de un programa de gestión ambiental para la implementación de la norma ISO 14001 en un taller automotriz. Quito - Ecuador: Trabajo de Grado - UNIVERSIDAD INTERNACIONAL DEL ECUADOR.

Díaz, R. A. (2011). Clasificación e identificación de materiales peligrosos. CEPIS/OPS, Curso de Autoinstrucción en Prevención, Preparación y Respuesta para Desastres por Productos Químicos.

Garzón-Jiménez, C. \&.-H. (2008). Inmovilización microbiana: técnicas y usos en el tratamiento de residuos tóxicos. Revista Sistemas Ambientales, 2(1), 23-34. 
Claudia V. Romero-Castro; Mónica M. Solórzano-Vélez; Adriana I. Navarrete-Ríos; Karen X. Córdova-Chinga; Iván A. Jaramillo-Chávez; Alejandra I. Navarrete-Ríos

Haddad, E., Serpa, R., \& Arias, R. (11 de Diciembre de 2018). IDENTIFICACIÓN Y CLASIFICACIÓN DE PRODUCTOS PELIGROSOS: CLASES DE RIESGO DE LA ONU, PANELES DE SEGURIDAD Y RÓTULOS DE RIESGOS. Obtenido de http://www.bvsde.paho.org/cursode/e/modulos/modulo_1.5.1.pdf

INEN. (11 de Diciembre de 2018). TRANSPORTE, ALMACENAMIENTO Y MANEJO DE MATERIALES PELIGROSOS. REQUISITOS . Obtenido de http://www.ambiente.gob.ec/wpcontent/uploads/downloads/2014/05/NTE-INEN-2266-

Lavell, A. (1999). Gestión de riesgos ambientales urbanos. Red de Estudios Sociales en Prevención de Desastres en América Latina. Red de Estudios Sociales en Prevención de Desastres en América Latina, 1-15.

Lavell, A. (2000). Desastres urbanos: una visión global. Woodrow Wilson Center and ASIES Guatemala publicación., 17-28.

Monge, G. (1997). Manejo de residuos en centros de atención de salud. Hojas de divulgación técnica, 69, 70., 1-12.

MSAL. (11 de Diciembre de 2018). Información general sobre sustancias toxicas e intoxicaciones. Obtenido de http://www.msal.gob.ar/images/stories/ministerio/intoxicaciones/emergenciasquimicas/

Muñoz Falconi, M. V. (2015). Manejo interno de los desechos infecciosos en las clínicas de octavo y noveno semestre de la Facultad de Odontología de la Universidad Central del Ecuador basado en el reglamento para el manejo adecuado de desechos infecciosos 2010. Quito Ecuador: Emitido por el Ministerio de Salud Pública del Ecuador. Trabajo de Grado UNIVERSIDAD CENTRAL DELECUADOR.

Neveu, A., \& Matus, P. (2007). Residuos hospitalarios peligrosos en un centro de alta complejidad. Revista médica de Chile, 135(7), 885-895.

Pública, S. (11 de Diciembre de 2018). Materiales tóxicos. Obtenido de http://pcchd.org/DocumentCenter/View/79/Materiales-Toxicos-PDF

Quinto-Mosquera, Y., Pérez, L. M., \& Arias, J. A. (2013). Conocimientos y prácticas de los trabajadores de un hospital sobre el manejo de residuos hospitalarios, Chocó, Colombia, 2012. Revista Médicas UIS, 26(1), 9-20.

Rodríguez Sordía, D. S. (2000). Desechos hospitalarios: aspectos metodológicos de su manejo. Revista cubana de higiene y epidemiología, 38(2), 122-126.

Sánchez, R. (1990). Manejo transfronterizo de residuos tóxicos y peligrosos: una amenaza para los países del tercer mundo. Frontera Norte, 2(3), 91-114. 
Schmidt-Hebbel, H. (11 de Diciembre de 2018). TOXICOS QUIMICOS EN ALIMENTOS. Avances en su identificación, previsión y desintoxicación. Obtenido de Universidad de Chile: http://repositorio.uchile.cl/bitstream/handle/2250/121416/schmidth09.pdf

Soto, F. O., \& Guerrero, C. (1996). Almacenamiento y manipulación de los residuos peligrosos. Journal of the Mexican Chemical Society, 40(5), 220-229.

UNAVARRA. (11 de Diciembre de 2018). MANUAL DE USO DE PRODUCTOS QUÍMICOS UPNA . Obtenido de http://www.unavarra.es/digitalAssets/146/146686_100000Manual-deuso-de-productosquimicos. 